\title{
Management Information Systems
}

\author{
Bogdan - Alexandru, FURDUESCU \\ Valahia University of Targoviste, 35 Lt. Stancu Ion Street, Building D, \\ bogdan_af@yahoo.com
}

\begin{abstract}
Technology is the science that studies processes, methods and operations run or applied onto raw materials, matters or data, in order to obtain a certain product. Information is the material signal able to launch a material reaction of a dynamic auto-tuning system for which the system is conditioned and finalized. Information Technologv is the technology needed for handling (procuring, processing, storing converting and transmitting) information, in particular, with the use of computers [Longley, D. \& Shain, M. (1985), p. 164]. The importance of IT in the economic growth and development is widely known, taking into account the impact that technology can have on the success and survival, or the failure of the economic activity of enterprises / organizations, IT offering various management information systems (MIS), executive and feedback segments, which all have important and beneficial implications in management and control.
\end{abstract}

Keywords: MIS purpose and role; MIS objectives; the main components of MIS; advantages and disadvantages of MIS.

JEL Classification: 015; J28.

\section{Introduction}

The way to conduct business in any organization is continually changing as a result of the combined action of the following external factors: globalization, competition at high-level, information (which became key resource), virtual work space, e-commerce and knowledge worker. The IT impact on the organization is felt not only from the external environment, but also from within of it. Any organization requires the existence of five interrelated elements (organizational structure, management and processes of the business, IT, organization strategy, employees and organizational culture) that must be kept in a state of equilibrium. This state will continue unless significant changes occur in the external environment or any of these elements. 
Appeared in the early ' 80 in the US enterprises / organizations, MIS are $a$ combination of human and computer resources aimed at collecting, storing, organizing, calling, communication, distribution and use of data and information that managers use in the performance of leading activities, in order to achieve an effective management, the impact of new information technologies and the flow of decentralization and reorganization resulting in increased demand for information from MIS at middle managers' level.

MIS are information systems designed to provide to the managers the information needed to monitor and to control business processes and to anticipate future performances. Offered in real time, this information is of particular importance for top-level managers, both for decision making in organization and for exercising an appropriate management control. The relevance of information brings together characteristics such as timeliness, accuracy and usefulness, and the MIS interface must meet the requirements of users who have little experience, limited skills in computer use or short time at their disposal.

There are three levels of MIS:

- operational management - planning and control of the daily operations;

- tactical management - planning and control at the level of organizational subunits;

- $\quad$ strategic management - planning and control of the entire organization, development of the organization strategy.

MIS must integrate the decision subsystem (management), the information subsystem (transmits / receives information to / from the decision subsystem) and the operational subsystem (in which the business processes specific to the activity domain are conducted) covering the information needs specific to each managerial level. 
Figure 1. Information and management levels

Unscheduled / Unanticipated

Synthesized

Random in time

Forecast character

Aimes at longer time horizons

It comes from outside of organization

Default content

Planned as the moment of obtaining

Detailed

High frequency of application

Punctual

Internal

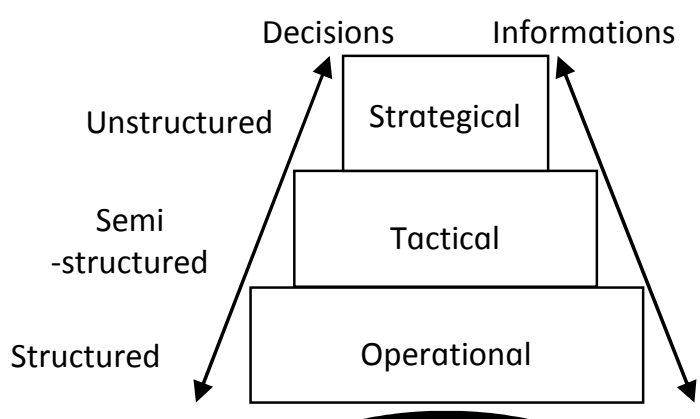

Management

Source: O'Brien, J. A. (1996), p.p. 32-38

\section{MIS purpose and role}

MIS is a set of interdependent elements which, based on default rules, establishes a dynamic interaction with the aim of achieving a particular goal. MIS purpose and role is to provide to managers easy and quick access to information about the key factors (decisive in achieving the organization's general objectives) and to allow them to exercise effective and efficient internal control management.

The first generation of MIS, characterized by the fact that they were proprietary and very expensive, was developed based on mainframes and retrieved data from the transactional information systems, simplifying, summarizing and graphically representing them, being focused on indicators for measuring the performance and on highlighting differences between budget or forecasts and actual results. The new MIS have a much broader usage, being installed on microcomputers or workstations from a network and bringing together information stored on a variety of platforms (mainframe, minicomputer or computers).

The purpose of MIS is to be as easy-to-use as possible, the response time to be immediate, to intensively use the graphically information display, to provide real-time access to internal and external databases, to provide information about current state of facts and expected trends for the key factors, the presentation 
form of information to be consistent with managers' preferences who are using the system. The information generated by computer not always has a leading role in the managerial needs, as for the CEO the most valuable information is the external one, which allows them to identify the organization's position on the global market and to analyze the external environment. Regarding the role, it can be stated that the MIS perform true functions, each one having several actions to achieve, namely: the prevision function (by deducting the information needed in a given situation, assuming the development of functional and dynamic forecasts based on the analyze of organization's history), the interpretation function (of information coming from the external environment which sets the system status), the processing function (of information through application and / or procedures regarding control data, creation of algorithms for working methodologies, generation of introductory and deductive procedures for selection of decision alternatives, development of interfaces between processes and applications), the diagnosis function (by capturing evidences of malfunction of system components), the training function (by familiarizing a wide circle of non-specialized users with dialogues in the format of hierarchy menus), the design function (of technical challenges and technologies) and the control function (specific to MIS through its own subsystem and interface monitoring).

Fgure 2. Management Information System (MIS)

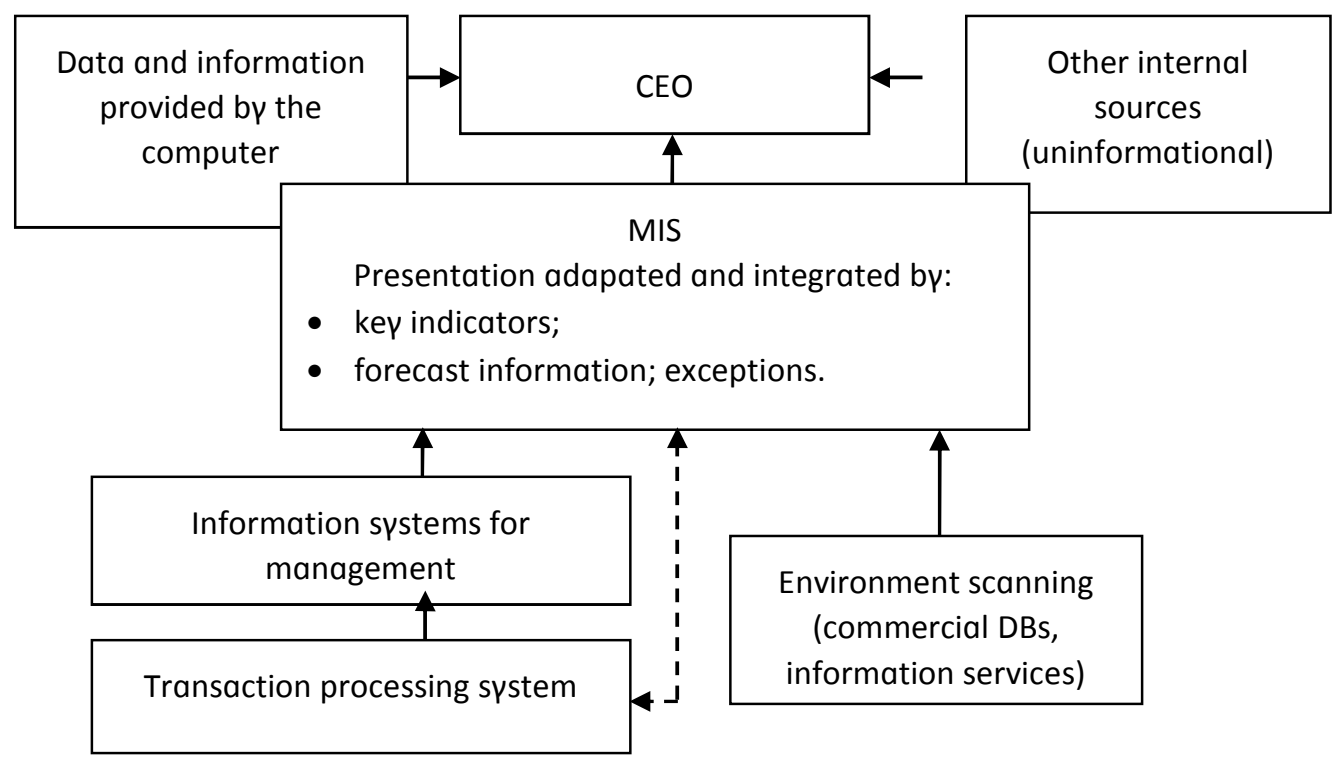

Source: Turban, E., McLean, E., Wetherbe, J. (1996), p. 45 


\section{MIS objectives}

MIS are characterized by the following: are intended for operational and tactical management, provide routine reports of synthesis type and deviation type (showing predefined structures), support the managers in solving structural issues (the made decisions have a routine character) and use mostly the organization's internal data (data provided by TPS). The MIS objectives can be structured as follows:

- $\quad$ managers' information about the activities and business processes from the organization, as well as its interaction with the external environment;

- $\quad$ timely access to information;

- $\quad$ monitoring the organization's performance and highlighting the aspects that hide potential problems or crisis situations.

By using MIS, the quality of information is obtained with minimum of printed reports (without using large amounts of paper) while providing a complete and permanent information to the leadership. The data selected to be included in MIS are based on the following principles:

- $\quad$ data must be collected from the organization's current processes that can be retrieved from databases developed by other information systems;

- data subordination to the organization's general objectives;

- $\quad$ provision of information to all potential users and their simulation simultaneously with the modification of information requirements.

MIS allow the access both to internal data and to external databases, as this is essential for decision making. also, MIS must support the "data mining" technique (the process of data provision for a specific component on multiple levels of detail for understanding the obtained results).

\section{The main components of MIS}

As with any information system, MIS main components are:

- Devices - are represented by microcomputers or networked workstations and minicomputers or mainframes. A station server (supports 
storage of large volumes of data) helps data sharing between multiple users. It is the most efficient architecture that optimizes existing hardware and software resources and adapt them to the requirements MIS; such architecture is the client / server one and uses SQL-based query language to provide solutions.

- $\quad$ applications - are the means of data handling and integration in the system, in which the word processor can incorporate search engines to locate and attract some parts based on kepwords.

- Database - is a tool for collecting and organizing the information, it is an "object container". The parts forming a database are tables, reports, queries, macros, modules. a database may comprise more than one table, one report, etc. a system for tracking the information about employees, products, orders or anything else is a database containing $\mathrm{n}$ tables, $\mathrm{n}$ reports etc.

- $\quad$ Spreadsheet programs and graphical tools - are environments adapted to the top-pyramid managers' requirements and style; they permit "What if ...?" analyses through the data modifying and the capability of independent data recalculation, the results can be obtained as tables or graphics.

- Model base - includes routine and special financial analyses, statistical analysis or other quantitative analyses, being aimed at structuring the analysis problem. The models included in the MIS must respond to strategic decision-making characteristics, different from what reflecting the tactical and / or operational management.

- Interface - provides human-computer communication and efficient operation of MIS. at present, the interface is intended to be adaptable to the top-manager's style, and in future it will be based on the natural language with the following characteristics: easy to use, consistency, clarity, fault tolerance and flexibility. 
Figure 3. Interfaces in MIS

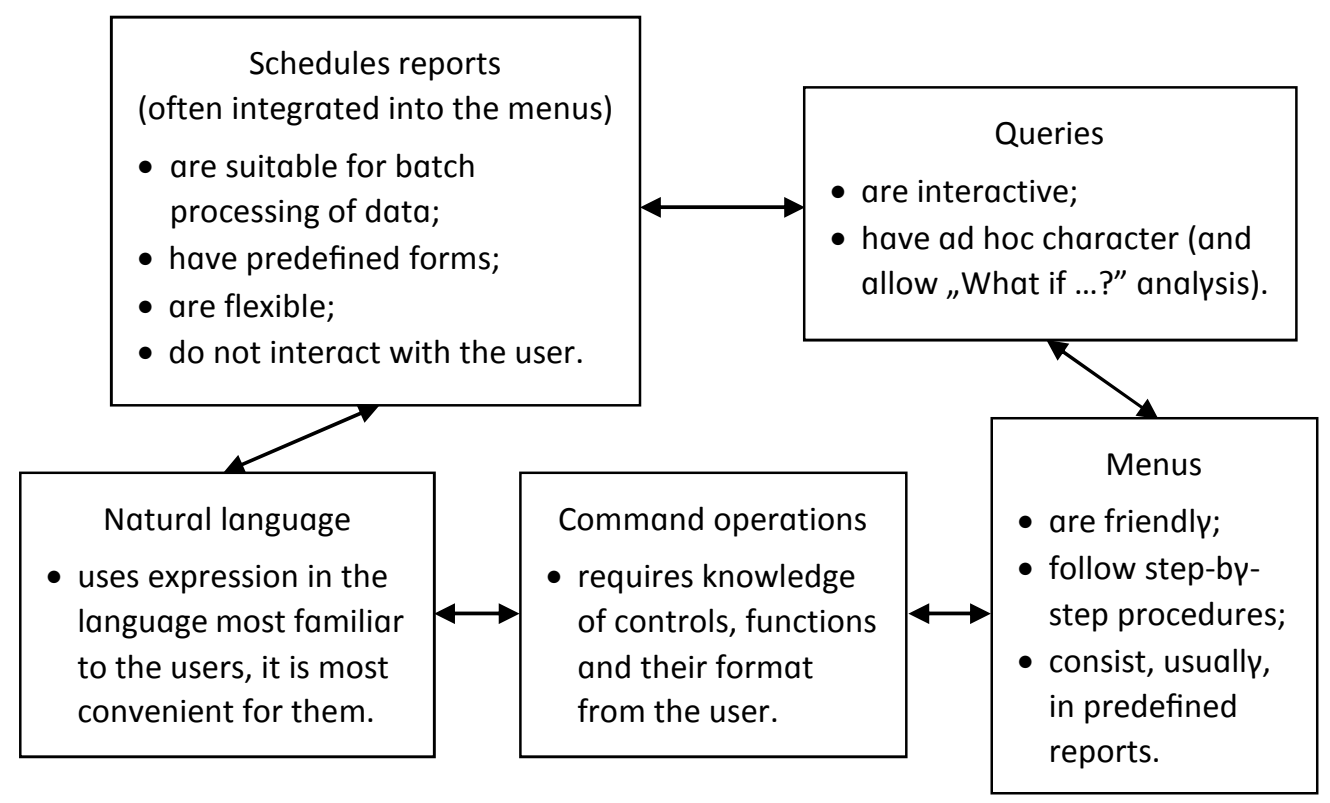

Source: The author

For efficient operation of MIS, the efficient combination of the hardware and software required to manage the data and models with a friendly interface is based on the criteria listed above, resulting a management control that is done in an efficient and performing way. A very important issue is the choice of software (specialized application package). The market offer of software dedicated to MIS is quite varied, and the choice of software should be based on criteria of: technical performance ("data mining" technique, reports of exceptions, easy-to-use interface, "What if ...?" analysis, capabilities of data consolidation and realization of multi-dimensional views on them, providing other applications on the interface), performance in application development (rapid development tools, easy updating of applications, rational database access and integration, graphical facilities), architecture (compatibility with existing equipment, flexibility, capability to integrate analytical online processing, integration with implemented databases), costs (with breakdown on equipment, applications, training, consulting, post-implementation), security 
and control (system security and data integrity). all these must be chosen so as to be compatible and to integrate with the situation from the organization.

\section{Advantages and disadvantages of MIS}

Unlike traditional MIS, the modern ones are characterized by the following: are tailored to the top-managers' needs information, provide consolidated reports and data on concrete issues, provide online analysis tools and exception reports and "data mining", perform information based on both internal data and those external of the organization, are easy-to-operate by using the mouse and / or the touch screen, needed special assistance is minimal, information is presented especially in graphical form.

The main advantages of MIS are:

- $\quad$ assurance of quality and timeliness of information needed by the management, without consuming huge amounts of paper, with a minimum of printed reports, while providing a complete and continuous information to the top-management;

- MIS are tailored to the top managers' information needs;

- MIS provide consolidated reports, and data on concrete issues and problems, detailed;

- provision of online analysis tools, including analysis of the prevailing trends, exception reports and data mining;

- performance of information based on internal data and also on external data of the economic organization;

- easy-to-use (based on graphical interfaces, operated with a mouse and a touch screen);

- $\quad$ needed special assistance is minimal;

- information is especially presented in graphical form.

Some of the MIS disadvantages are:

- $\quad$ insufficient knowledge of MIS possibilities by managers;

- need for special training of all categories of staff involved; 
- $\quad$ need to introduce into the system and operate quality and correct data;

- $\quad$ need to correlate the methods and techniques encapsulated in the processing and presentation of data in the system;

- $\quad$ mistrust of some MIS managers.

When MIS are implemented in organizations, there may appear some problems that lead to decreased efficiency of major interest activities, namely: lack of managers' proper involvement in MIS designing, inadequate attention paid to the possibilities offered by MIS, an inadequate level of concern granted to the applications of data processing at lower levels of management because of the managers' lack of depth information knowledge, a wrong assessment by computer scientists of managers' information requirements and specific problems of organization, lack of real and convincing support from the top-management.

\section{Results}

According to the systems' theory (an interdisciplinary epistemological model that uses systems to describe and explain phenomena of vary degrees of complexity), any economic body is a system (a set of interdependent elements / components among which a dynamic interaction is established, based on predefined rules, in order to achieve a certain objective) because: it has its own structure consisting of a set of constituent elements that interact with each other based on functional principles, the flows among organizational components use resources of the economic bodv and organizational components, and their interactions aims to attain a certain overall goal, namely the functioning of the organization in optimal conditions or the achievement of certain objectives.

MIS is a combination of human and computer resources that seek to collect, store, organize, request, communicate, distribute and make use of data and information that managers use during their activities in order to provide an effective management. This system plays a dual role: on one hand it provides all necessary information for decision-making at all levels of responsibility, leadership and control, and on the other hand it provides means of communication among other systems, decisional and operational ones, because decisions made by the decision system are transmitted to the operating factors through the information system (downstream). Thus, MIS represents the entirety 
of the processes, methods, techniques and means of collecting, transmitting, processing, storing and archiving information necessary for decision making, the main purpose of MIS being to provide each user all necessary information, according to his / her responsibilities and attributions.

\section{Discussion and Conclusions}

MIS represent a category of information systems designed to increase economic efficiency, to raise the growth potential of the business, to perform a more efficient and better control, to support and improve the decision making. The new management strategies based on the implementation of management information systems have caused profound changes in the organization's organization and functioning, of which the most important are: minimizing the number of organization's hierarchical levels, appearance of preferences for flexible organizations, generation of virtual organizations and elimination of distances between different links from the chain of organizational management, electronic business development (especially e-commerce) and reconsidering the structure of document flows, meaning the transfer from paper support to electronic support.

\section{References}

[1] Buse, F. (1998). Informatica manageriala. Deva: Ed. Calauza.

[2] Damidonciu, a., s.a. (1995). Sisteme de inteligenta artificiala. Bucuresti: Ed. Economica.

[3] Longley, D., Shain, M. (1985). Dictionary of Information Technology $\left(2^{\text {nd }}\right.$ ed.). London: Macmillan Press.

[4] O'Brien, J. a. (1996). Management Information Systems: Managing Information Technology in the Networked Enterprise ( $3^{\text {rd }}$ ed.). Chicago: Irwin.

[5] Turban, E., s.a. (1996). Information Technology for Management: Improving Quality and Productivity. New Гork: Whiley\&Sons.

[6] *** (1999). Tribuna Economica, nr. 40. 